\title{
Extending Web educational applications via SGML structuring and content-based capabilities
}

\author{
B. Fernandez-Manjon, A. Fernandez-Valmayor and A. Navarro
}

Escuela Superior de Informatica,

Universidad Complutense de Madrid, Avda. Complutense s/n, 28040, Madrid, Spain

e-mail: bfmanjon@dia.ucm.es,valmayor@fis.ucm.es, anavarro@eucmax.sim.ucm.es

\begin{abstract}
In this paper we present an approach to the development of World Wide Web educational applications based on a deeper understanding of the role that mark-up languages play in the Web, and how they could improve Web educational applications. The paper begins with a discussion of the Web's key features that have made it so successful. Then we present and analyse different approaches to educational uses of the Web. We introduce the SGML/HTML as a tool to organise and structure information, and propose a new approach to the development of Web educational applications based on SGML extensions to the HTML language. Finally, we use our own experience in developing educational applications as an example of the new possibilities offered by this approach.
\end{abstract}

Keywords

Web applications, Mark-up languages, Educational multimedia, HTML, SGML 
Initially, the Word Wide Web was designed as a researchers' tool for sharing information and producing documents in a collaborative/co-operative way over the world's largest computer network, the Internet (Berners-Lee, 1994). However, the Web is now the fastest growing resource on the Internet. It is not difficult to understand why the Web is growing exponentially and why it is used by all kinds of institutions and people in all type of activities: academic, commercial, entertainment, etc. All of them find advantages in the way the Web can manipulate information, that is: simplicity of use, an attractive presentation, an easy way to relate dispersed information, low cost of creation and maintenance of the information nodes, and finally, facilities for interaction and communication. What it is maybe not so evident to Web users is that all these characteristics that make the Web so flexible and attractive are based on a mark-up language defined using a complex standard, the SGML (Standard General Mark-up Language). Browsers can interpret documents written using an application of this language: the HTML (Hypertext Mark-up Language) documents or Web pages. Therefore, it is the definition and interpretation (i.e. the syntax and semantics) of this language which set the limits of what it is possible to do in the Web, and it is SGML the main tool we should use if we want to change those limits.

From an educational point of view and due to the increase of information available (and the Web is a main contributor to this information explosion) new ideas are breaking through, and educators are reconsidering traditional approaches to education in the new light provided by the information technology. Now, viewing education as the mastery of a body of knowledge is becoming outdated. Instead, we recognise that students need more than ever to have acquired skills, such as rapid comprehensive reading, critical thinking or qualitative reasoning, along with abilities, such as the ability to find needed information and the ability to work well with others (Twigg, 1994).

Many educators (Jonassen, 1995; Trentin, 1996; Wulf, 1996; Ibrahim, 1995) consider that information technology, and specifically the Web, have to play a central role in those educational changes, driving the information explosion and making possible the construction of new learning environments. But the key to the successful use of the Web as an educational environment is a deeper understanding and clarification of its possibilities and possible roles in education. There is no doubt about the benefits the Web can bring to education: co-operative/collaborative work and the remote and egualitarian access to information are often mentioned as the benefits of networking and Internet (Collis, 1995), but to share information is not to educate and having access to information does not imply learning. At present, there are still some technical problems (e.g. the limited capacity of the communication link, slow modems access), but these problems are not our main concern because these technologies are improving all the time. The real problem is how to deal with cognitive and pedagogic situations in the Web. In part because 
the Web was not originally designed as an instrument to educate people problems like disorientation, lack of planning or guidance on the access to information, and information overload affect more seriously the usefulness of the Web as an educational tool (Buenaga, 1995; Nunes, 1996).

This paper is organised as follows. First we present and analyse current educational applications and proposed taxonomies on educational uses of the Web. Then we propose new approaches to Web uses in education. We also present the HTML and investigate in which way it is possible to use it or its proposed extensions as an educational tool.

\section{WEB EDUCATIONAL USES: TAXONOMIES}

Entering the Internet, from a computer at home or at workplace, users can access a vast amount of library catalogues, course materials, journal indexes, reference books, full text of journal articles and books, art exhibits, employment notices, discussion groups, business data, etc. Clearly, the availability of all this information offers us new opportunities for teaching and learning. Consequently, there has been a growing interest in the educational uses of the Web. Hence to better explore the potential role of the Web in education, we will start analysing some of the proposed taxonomies on these educational uses of the web.

The taxonomy offered by Wulf could be representative of those classifications that take into account the purpose of use (i.e. for what we are using the Web). Wulf (1996) identifies seven different uses of the Internet in education:

1. Electronic mail: delivery of course materials, sending in assignments, getting/giving feedback, using a course electronic discussion group.

2. Bulletin boards/newsgroups for discussion of special topics.

3. Downloading of course material or tutorials.

4. Interactive tutorials on the Web.

5. Real-time interactive conferencing using systems like Internet Relay Chat.

6. "Intranets": corporate websites protected from outside access that distributes training for employees.

7. Informatics: the use of on-line databases, library catalogues, and gopher and websites to acquire information and pursue research related to a study.

Other classifications put the stress on the way that educational applications take advantage of Web attributes like interconnectivity or the presentation and interrelation of information. In this sense, Carvin (1997) identifies four different educational uses of the Web:

1. The Web as tutor: that is, the Web as a way to provide on-line tutorials and lessons, based on its ability to present information clearly, attractively and practically.

2. The Web as publishing house, so teachers and students can create in-depth "hyper-reports" with links leading to numerous subtopics and related network connections. 
3. The Web as forum for discussion of ideas and virtual debate, for example using mailing lists (listserv).

4. The Web as navigator, or as a tool to search for information all over the network.

On the contrary, other authors claim that the classification of use in education of computer networks has to be done taking into account the degree of enhancement (added value) offered by this technology and not so much by its particular uses. The main difference is between situations, or contexts, in which innovation brought to them by telematics is mainly a technological improvement and new situations that could not take place without the network. Examples of the first case, of technological improvement, is the handling of intercommunications via electronic mail or the remote access and sharing of data and educational materials. Examples of the second case, new situations, are co-operative learning with on-line interaction in virtual classrooms. That way, Trentin (1996) identifies three educational contexts:

1. Plain utilisation of the network for communication, based not so much on specific approaches, but rather on using the network to provide a powerful tool for navigation through distributed information and for interpersonal communication.

2. Using computer networks in support of educational activities that can be conducted with or without the network but which in this way are improved producing greater motivation and involvement.

3. Learning activities based on specific approaches that are strictly dependent on the use of specific characteristics of the network and could not exist without it.

Finally, it is also necessary to notice that there are too many specific applications and research projects using the Web in educational settings to be possible to do a comprehensive study. For instance, Computers \& Education (Kibby, 1995), and Internet World (1995) special issues about Education and Internet present some particular uses. General discussions about the roles of telematics and new hypermedia technology in education can be found in (Jonassen, 1995; Kearsley, 1995; Davis, 1995; Laurillard, 1995; Nunes, 1996; Kerka, 1996).

\section{NEW APPROACHES TO WEB EDUCATIONAL USES BASED ON WEB LANGUAGE CAPABILITIES AND SGML EXTENSIONS}

At first it seems natural to think that with all these characteristics the Web can be the ultimate educational tool. Nevertheless, the first conclusion of our survey is that most applications and research analysis as those previously mentioned take for granted a positive effect when using the Web as an educational resource. However, the outcome of our survey is that even if most of the projects dealing with the educational possibilities of the web come to mainly positive conclusions, in these projects it is not clearly stated and defined which are the unique characteristics in 
the educational activity. Moreover, in general the requirements of the new educational frameworks that the web could help to develop are not described.

We consider that the key characteristic of the Web is its mark-up language. It is HTML that converts a document into a hyperdocument. This hyperdocuments can contain appealing multimedia information, and can be accessed remotely by browsers. Therefore, we think that a taxonomy of web educational uses have to be based on the contributions that HTML, or more generally, SGML could bring into education together with improvements in communications technology. We would try to synthesise those classifications in a comprehensive one. In the first place, we would also distinguish between the educational context where the innovation brought by the Web is mainly technological and new contexts promoted by the Web that could not exist without it (Ibrahim, 1995).

1. The Web as a technological improvement, enhancing human communication and human access/sharing of information.

- Enhancing human to human communication in a distance learning or educational context (e.g. e-mail and list-servers).

- Student/student.

- Student/teacher.

- Teacher/teacher.

- Enhancing human access to educational objects (e.g. downloading courses and tutorials).

- Enhancing human access to information objects in an educational context (e.g. databases, repositories).

2. New situations based on specific characteristics of Web's language that offer a new kind of learning situations to students.

- Interacting with Web based tutorials that could need or not specialised viewers or browsers. A web-based tutorial will not only use HTML tags for hypertext and hypermedia links but will also use HTML tags to pedagogically structure the document.

- Interactive searching of information in the Internet. That means not only that students and teachers can use search engines (e.g. Infoseek or Lycos), but also they systematically make use of previous efforts of other users reflected in well-organised personal pages.

- Personal or collaborative building of well structured Web documents and/or Web based tutorials.

- Virtual classes in open and more authentic contexts that promote a meaningful learning (Jonassen, 1995). The web is not merely used as an information delivery medium, but rather as an organiser of thoughts and facilitator of knowledge construction.

This double classification tries to state the singular role of the mark-up language in the Web. In the applications comprised in a) the HTML language is used to integrate different operations in a simpler and friendly interface. These operations can be done using different Internet tools like ftp, e-mail, IRC or telnet. But, the 
applications comprised in b) are based in a more educational approach to the possibilities offered by HTML. For example, from a web tutorial it is equally simple accessing a local document as a remote document or even to look for complementary information in a different server.

Nevertheless, we think that today most educational applications do not take full advantage of the (limited) possibilities offered by HTML. The role of the web seems to be restricted to hypertextbook type applications where the predominant model continues to focus on the production of information resources and much less on the learning processes in which the student must be engaged. Possible reasons for the present situation are:

- Current HTML definition has been done taking into account mostly the needs of the net's publishing industry requirements (i.e. mark-up for tables, styles, forms, etc.).

- There has not been a substantial development of tools, browsers and editors, specifically adapted to educational tasks.

Therefore and as a result of our analysis we conclude that there are two main lines for future work and research in order to transform the Web into a more powerful educational medium:

- First, to develop special browsers for a generalised mark-up language able to capture the logical structure of a document, beyond simple characteristics such as title or body text. These browsers could be an intermediate step between a generalised marked document and a HTML document or a full marked document browser (Burnard, 1997; Sperberg-McQueen, 1994).

- Second, to provide the specifications and requirements to develop help and instructional tools that make use of the educational possibilities of the language. Here we identify two possibilities: a) Tools that help to create and interpret Web pages specifically created with a pedagogical purpose; and b) Tools that process generic Web pages with educational purposes.

Finally, we have no doubts about the benefits and potential of the Web in educational institutions, and specifically in those providing distance education. Perhaps more than any other previous media, the Web can help to overcome the barriers of time and space in teaching and learning (Kerka, 1996). But, it is necessary to notice that the educational value of the Web is also limited. The Web will not be the solution to all educational problems. Therefore, it would be very positive to identify clearly the situations where the Web could be used to solve educational problems. 


\section{ORGANISING AND STRUCTURING EDUCATIONAL INFORMATION USING HTML/SGML}

As previously stated, we think that most educational applications are not exploiting fully the possibilities offered by the present HTML. Web pages are structured documents which contain mark codes, that can not only be used to format documents for on-line presentation but also as a way to promote good cognitive skills in the learners. In order to obtain a really useful educational Web it would be necessary to help the learners to convert the information into knowledge avoiding problems like information overload, for instance.

The mark-up can identify portions of a document and can be a means of making explicit an interpretation of its actual content. Thus, we envisage the use of the HTML as a way to add value to the information provided in the Web. We consider the HTML language as a tool that can help to organise and structure the content of a document according to pedagogical ideas. This consideration will permit the application of pedagogical strategies in the presentation of the information making the Web a suitable tool to ease the process of learning.

The Web is written largely in HTML, which is one application of the Standard Generalised Mark-up Language (SGML). The explosive growth on the World Wide Web applications demands richer and larger sets of mark-up features in HTML. SGML introduces new concepts in information handling and exchange of information at all levels of complexity. Newer revisions of HTML will include greater numbers of SGML features. As the Web continues to grow, SGML will likely become the standard for more mark-up languages on the Internet (Colby, 1996).

\subsection{Introduction to mark-up languages and their applications}

The Standard Generalised Mark-up Language (SGML) is a meta-language that can be used for encoding the logical structure and content of any type of document. The goal of SGML is to standardise the definition of device-independent, systemindependent methods of representing the structure and intent of a document, so the accessibility and reusability of information stored within documents can be maximised. Since 1987, SGML has been widely adopted by government, industry and academic groups world-wide.

SGML is not a set of standardised codes but a language that can be used to define precisely the elements to which a particular kind of document must conform. This definition starts by an SGML declaration, which states mandatory and optional features, used in the rest of the definition. This declaration is followed by the document type declaration (DTD) which is the formal collection of element, attribute and entity declarations that describe what mark-up to expect in this kind of documents. Finally there is the document instance, that is the document itself with the actual mark-up. The real benefit of this flow is that a computer program, a SGML parser-translator, can process the declaration and learn its rules, then 
process the DTD and learn the rules of the mark-up, and then process the document and determine if the document meets the rules and take the prescribed actions. This way an SGML document can be processed by different programs and with different purposes; each program can apply different processing instructions to those parts of the document instance, which are considered relevant.

Part of the possibilities of SGML are already being used in educational projects like the Text Encoding Initiative (TEI) and the International Committee for Accessible Document Design (ICADD) (SGML Open, 1996). ICADD leverages an SGML tagged document to produce multiple textbook versions from the same source document, giving print-disabled students access to the information (through Braille, large print, voice synthesis, and electronic browsing). TEI is an international effort to standardise encoding of all kinds of text in any language (mainly literary and historical texts). TEI encoded documents give researchers and students an efficient way to utilise the information contained in these texts. As these and other new initiatives evolve, more information can be handled more efficiently giving educators and students a way to manage the voluminous quantities of available information.

The web language, HTML, is an SGML application and as such its syntax is defined by the combination of the SGML declaration and the document type definition or DTD. Actually, the current HTML definition in force, HTML 3.2, places further constraints, inexpressible in the DTD, on the permitted syntax, although HTML is moving towards the larger feature set of SGML. Consequently, more SGML tools are starting to appear for the average users. For example, there is a SGML Web browser that works alongside a standard browser (e.g. Netscape) (Colby, 1996). When you encounter an SGML Web page, the program activates and enables you to browse it as easily as an HTML page.

Therefore, HTML has the potential of being a really smart use of SGML, taking advantage of SGML's flexibility without giving up a common backbone structure that any browser can readily interpret (Severson, 1995). Moreover, it is precisely this combination of simplicity and extensibility that will enable the Web to be viable for new educational applications.

\subsection{A basic example of SGML application to information structuring}

In this section we use a hypothetical DTD that represents a simple fax type document, along with one real fax to illustrate some of the possibilities offered by SGML. A full discussion of the SGML is out of the scope of this paper and can be obtained elsewhere (Goldfarb, 1990, Colby, 1996).

In a document we must distinguish: structure, content and format. The structure is the document's logical organisation. Content is the actual data in a document. Format consists of how the elements are visually presented and distinguished from one another within a document. 

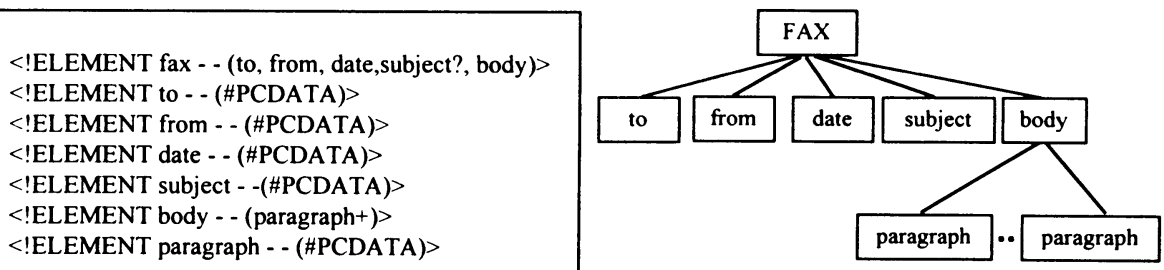

fax.dtd

Figure 1 Fax DTD and its tree representation.

To obtain the content model or the outline structure of a fax, it is necessary to identify which are the important parts (called elements) that made up a fax. In our fax these elements are: the origin, the destination, the date, the subject of the message (that we will consider optional) and a body (the actual message) made up by one or more paragraphs. SGML captures the fax structure using a DTD and it can be visually presented as a tree (see Figure 1). We can understand a DTD as a grammar (i.e. the set of rules) to whom a document must conform. The structure defines how the document is laid out, which elements can be required or optional, if an element is repeatable or not, and in what order the elements are assembled.

In the DTD we define the tags used to mark the elements of the real document. The boundaries of an element will be given by the star-tag and end-tag. By default, SGML tag mark-up consist of the name of the element type surrounded by angle brackets $(\diamond)$, with the addition of a slash $(/)$ before the name of the end-tag. For example, the body of the message is enclosed between the tags <body $>$ and $<$ /body $>$. \#PCDATA means that an element contains characters.

A real fax is represented via a document instance or marked document. In the marked document we employ the DTD tags to sign the document parts. For example, for the fax instance, the mark-up would look as follows (some elements are shown on separate lines and indented with the content in bold face for ease of reading, but it is not necessary):

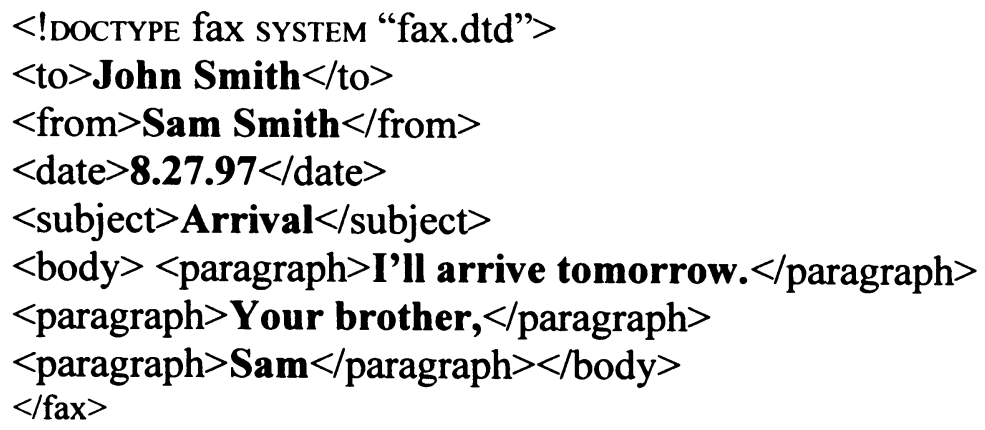


Because we have the fax represented by two parts, the DTD and a document instance, we can perform different treatments of the fax, maintaining the difference between structure, content and presentation. An important treatment is document rendering being possible to produce multiple sets of formatted output from the same document instance. But manipulations are not limited to rendering as in HTML. On the contrary, SGML also allows the automatic validation of a document checking its structural validity according to the rules of the DTD (e.g. if the fax contains the destination -element to- that is mandatory, or if at least one paragraph appears in the body). Moreover, it is possible to process and search for text based on its context, such as to search a fax containing a specific text in the subject or select those faxes that have more than 200 words in the body text. SGML also includes facilities called attributes to further describe the content of the elements and to establish cross-reference mechanisms. For instance, the fax DTD could include an attribute describing the type of the fax as personal or business.

Thus, we think SGML is the way to obtain richer mark-up languages that we needed to develop new Web educational tools. SGML is able to capture all document facets, providing us a way to design, develop and maintain new educational tools.

\section{TEACHING TEXT COMPREHENSION IN A SECOND LANGUAGE: THE GALATEA PROJECT}

Included in the SOCRATES/LINGUA European Union project, our group is working on the Galatea project with other European universities under the Stendhal-Grenoble III University leadership. This project is aimed at developing a set of multimedia tutorials for the written and oral comprehension of the Romance languages (see Figure 2). The pedagogical and cognitive strategies included in the set of multimedia tutorials being developed by our group are based on previous work about text comprehension done by group's members (Lopez, 1994; Fernandez-Valmayor, 1992) and also taking into account ideas of others authors (Schank, 1977; Kintsch, 1978; Adam, 1992; Grosz, 1995).

We take our work in this project as an example to describe the different roles that the Web can play in an educational application. The pedagogic scenario used in the tutorials we are producing is based on the selection of about 10 different documents written in French and in a sequence of exercises that students can make on each of these documents to improve their comprehension level of the written text. The main goal is to promote the understanding of the document content even if some words or paragraphs are initially incomprehensible. There is also a set of general-purpose tools that students can freely use: a general dictionary, a contextual dictionary, a contextual grammar and a sound tool to hear the texts. The general learning strategy is to relate the information contained in the document with information previously known by the student (Ausubel, 1963). For instance, an exercise can ask the learner questions aimed at the correct classification of the text based on external traits easy to recognise (e.g. a journalistic text, an 
advertising text or a fiction text). Other exercises take into account the temporal script: the prototypical sequence of events implied by the main topic of the document, (e.g. the sequence of events in a fire), or the set of words belonging to the same semantic group (e.g. words related to a fire, or words related to the family -as shown in Figure 3-). The student has the assistance of general-purpose tools, such as a dictionary and specific tools related with the specific examples appearing in the tutorial such as the contextual grammar and the contextual dictionary called "paso a paso" (see Figure 4).

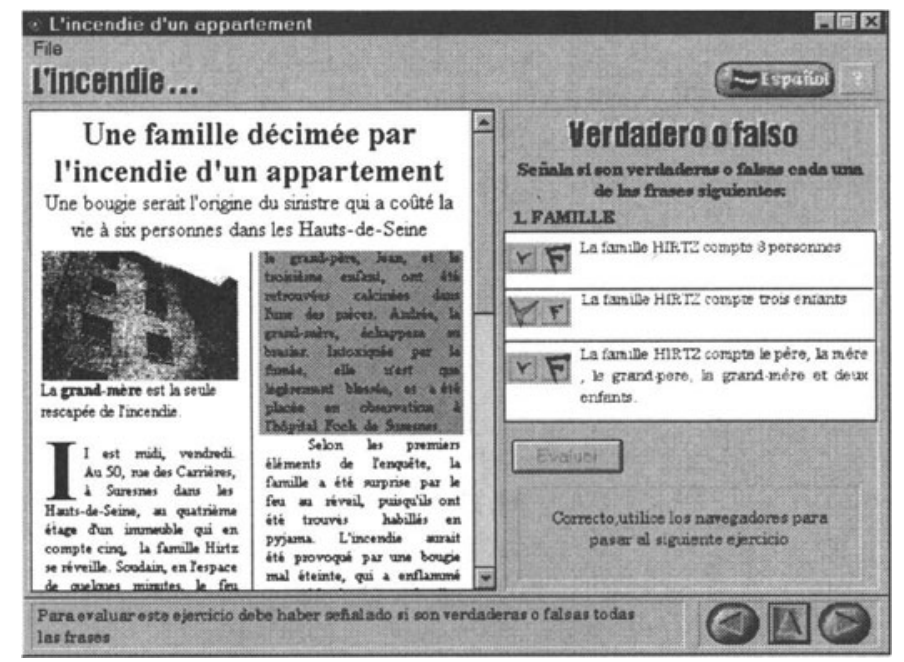

Figure 2 Screen of the Galatea current multimedia application for teaching French text comprehension.

On the one hand this kind of tutorial could be considered as a finished product that can be distributed over the network for remote execution, or for download and local execution. But on the other hand we think that there is a more promising approach making use of the same capacities on which the Web is based. This approach is to provide the documents with all the mark-up information necessary to implement general educational strategies similar to those used in our tutorial. The particular strategies used to present the information will be interpreted by a specialised browser able to understand the mark-up of those documents or, to a limited extend, these strategies could be included in a program for translating those SGML marked documents into HTML documents to be later viewed with a general browser.

Providing the mark-up documents facilitate on the creation of new educational applications because the presentation strategies of the information can be changed or adapted locally. For instance, this approach can simplify the automatic creation of a comprehensive content summary of a Web document based on its marked 
information. This approach can also simplify the sharing of documents and even the collaborative construction of repositories to be used in text comprehension.

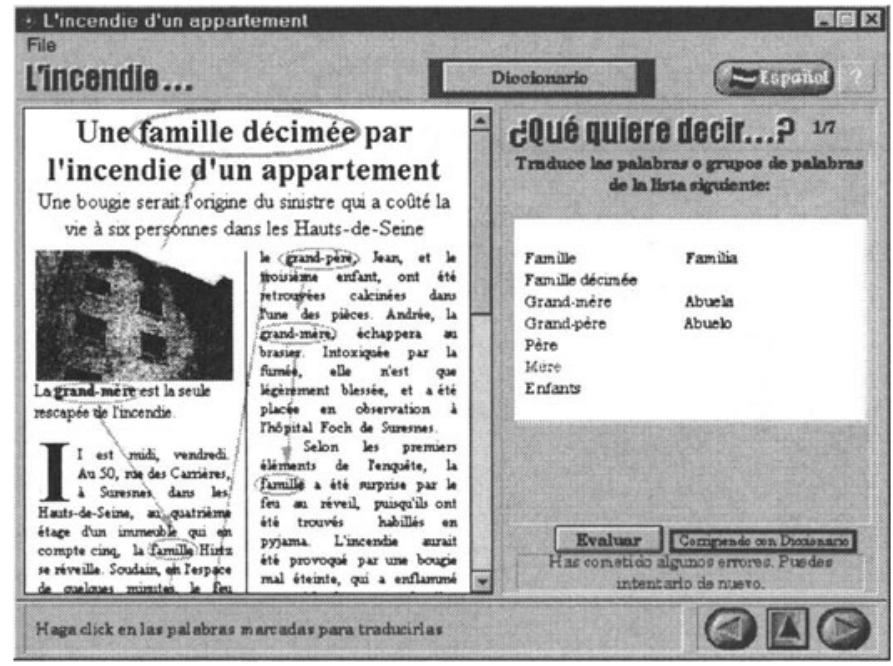

Figure 3 Exercise to improve comprehension by the translation of the set of words related to the family. After the evaluation of user's answers the user can access the dictionary to look up the meaning of the words.

Now we are shifting our approach because we realise that the use of SGML techniques is not only restricted to the mark-up of texts (i.e. to capture the information about structure and content) to be later viewed with a specialised browser (i.e. to decide the final rendering) but also they can be fully applied in the application design and maintenance steps, closing the gap between designers and programmers. This is possible because we can use a SGML DTD to capture the structure and some of the design decisions that are at the base of the educational tool under development. For example, in the texts we need to mark different kinds of information such as the keywords or several sets of related words that the user have to understand in order to get the meaning of the text. These different elements used to teach text comprehension are described in the DTD and using this DTD labels we mark the keywords or the set of families appearing in the text. Therefore the combination of the DTD plus the marked texts provided much of the design information and pedagogical decisions of the educational application.

\section{CONCLUSIONS AND FUTURE WORK}

The development of our educational application has showed us that the present definition of HTML is insufficient to mark-up and organise all the data contained in the documents that are relevant to our application. Thus we are now working in 
an extended mark-up using SGML. But in many of the current Web browser, any mark-up not recognised as supported HTML element is ignored. So this new extensions could be only interpreted with new browsers specifically designed for educational purposes or with new add-on programs (specialised parsers) for standard browsers.

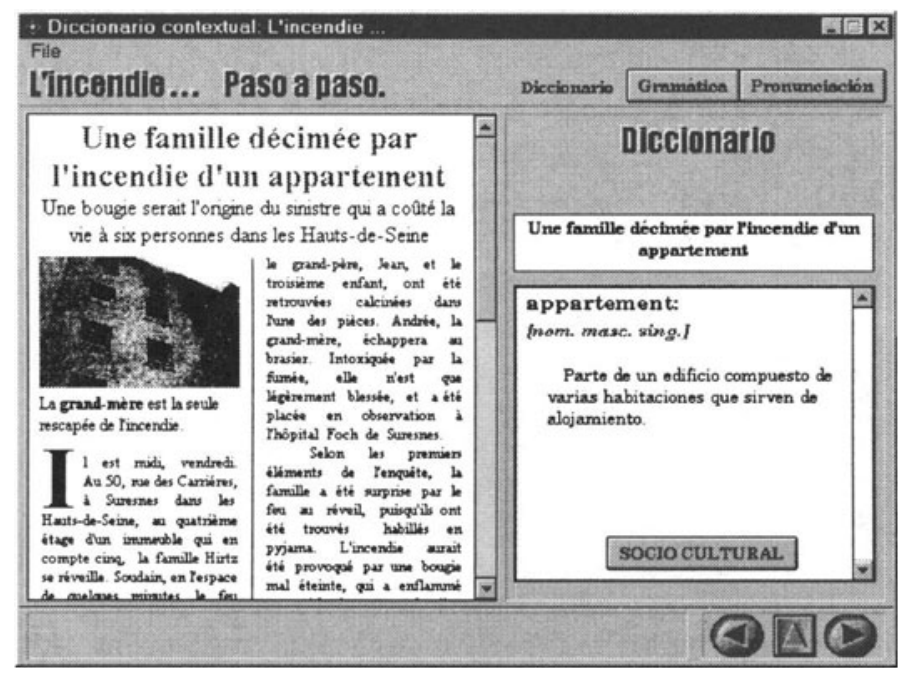

Figure 4 The user can also access a contextual dictionary where complementary information about words is provided.

We realise that a powerful communication resource like the Internet can provide new incentives and unprecedented opportunities for innovation in the field of education. The crux of the matter is to define innovative methods for designing, planning, and conducting network actions in which innovation lies not just in the mere presence of a new technology but rather in revising certain teaching processes or in creating new ones based on the new technology (Trentin, 1996). The Web is accepted internationally because its ease of use, capabilities for presentation/communication and cross-compatibility and future changes in HTML standards will make it even more powerful. It would be desirable that the future HTML standard also included capacities for educational use and not only those interesting to publishers (e.g. better tables or layouts). In other case, if we want to be able to develop and maintain complex applications extending Web educational use we should migrate to SGML or SGML/HTML browsers.

The next steps of this research will be to produce Web applications for text comprehension. At present, we are working on the final completion of the (local) multimedia educational modules for second language text comprehension (French). Currently in these modules we are using specific mark-up that we are translating to SGML format. In the future these tags will also be used for the contextual dictionary. This dictionary will consider not only the definition of words and text 
segments, but also its features (morphologic, syntactic and semantic) and even its use in specific text examples (see Figure 4).

Long-term objectives of the project are to obtain an educational DTD for teaching text comprehension and the use of SGML technology to simplify the design and maintenance of educational applications.

\section{AKCNOWLEDGEMENTS}

The EU project Galatea (TM-LD-1995-1-FR89) and the Spanish Committee of Science and Technology (TIC96-2486-CE) have supported this work.

\section{REFERENCES}

Adam, J.M. (1992) Les textes: types et prototypes, in Recit, description, argumentation, explication et dialogue. Nathan, Paris.

Ausubel D.P. (1963) The Psychology of Meaningful Verbal Learning. Grune and Stratton, New York.

Berners-Lee, T., Cailliau, R., Luotonen, A., Nielsen, H.F. and Secret, A., (1994) The World Wide Web. Communications of the $A C M, 37(8), 76-82$.

Buenaga, M., Fernandez-Manjon, B. and Fernandez-Valmayor, A. (1995) Information Overload At The Information Age, in Adults in Innovative Learning Situations (eds. B. Collis and G. Davies), Elsevier Science B.V., Amsterdam.

Burnard, L. (1997) SGML on the Web: Too Little Too Soon, or Too Much Too Late. Computers \& Texts, 15, August.

Carvin, A. (1997) The World Wide Web in Education: A Closer Look (WWW document, URL: http://k12.cnidr.org:90/web.intro.html)

Colby, M. and Jackson D. (1996) Special Edition Using SGML. QUE Publisher.

Collis, B. (1995) Societal and organizational influences on integration: what about networking?. In Integrating Information Technology into Education (eds. D.Watson and D.Tinsley), Chapman \& Hall, London.

Davis, N. (1995) Distance Education with Telematics for Distance Educators. International Journal of Educational Telecommunications, 1(2/3), 199-211.

Fernandez-Valmayor, A. and Fernandez, C. (1992) Educational and Research utilisation of a Dynamic Knowledge Base. Computers \& Education. 18(1-3), 51-61.

Goldfarb, C.F. (1990) The SGML Handbook. Oxford University Press.

Grosz B.J., Joshi A.K. and Weinstein S. (1995) Centering: A Framework for Modeling the Local Coherence of Discourse. Computational Linguistics 21(2), 203-225.

Ibrahim, B. and Franklin, S.D. (1995) Advanced Educational Uses of the World Wide Web. Computer Networks and ISDN Systems, 27(6), 871-877. 
Ide, N. and Veronis, J. (1995) Text Encoding Initiative, Background and Context. Kluwer Academic Publishers, Dordrecht.

Internet World (1995) The Internet in Education. Internet World, Special Issue, October 1995, 38-85.

Jonassen, D.H. (1995) Supporting Communities of Learners with Technology: A Vision for Integrating Technology with Learning in Schools. Educational Technology, July-August.

Kearsley, G., Lynch, W. and Wizer, D. (1995) The Effectiveness and Impact of Online Learning in Graduate Education. Educational Technology, NovemberDecember.

Kerka, S. (1996) Distance Learning, the Internet, and the World Wide Web. ERIC (Educational Resources Information Center) Digest, ED39521496, Columbus, Ohio.

Kibby, M.R. and Heller, R.S. (1995) Education and the Internet. Special Issue, Computers \& Education, 34(3).

Kintsch, W. and Van Dijk, T. A. (1978) Toward a model of text comprehension and production. Psychological Review, 85, 363-394.

Laurillard, D. (1995) Multimedia and the changing experience of the learner. British Journal of Educational Technology, 26(3), 179-189.

Lopez Alonso, C. (1994) Comprehension et texte: role des cadres de connaisances et du prototype. Langages. Theorie et applications en F.L.E., 157-174.

Nunes, J. and Fowell, S.P (1996) Hypermedia as an experimental learning tool: a theoretical model. Information Research New, 6(4), 15-27.

Schank, R.C. and Abelson R.P. (1977) Scripts, plans, goals, and understanding. Hillsdale, Lawrence Erlbaum Associates, NJ.

Severson, E. (1995) How SGML and HTML really fit together: The case for a scalable HTML/SGML Web. (WWW document, URL:http://www.ileaf.com/avhome/htmlscal.html)

SGML Open, (1996) SGML in Education: The TEI and ICADD Initiatives. Computers in Libraries, 16(3), 26-28.

Sperberg-McQueen, C. M. and Goldstein, R. F. (1994) HTML to the Max: A Manifesto for Adding SGML Intelligence to the World Wide Web in Proceedings of the Second World Wide Web Conference '94: Mosaic and the $W e b$, Chicago, IL.

Trentin, G. (1996) Internet: Does It Really Bring Added Value to Education?. International Journal of Educational Telecommunications, 2(2/3), 97-106.

Twigg, C. A. (1994) The Changing Definition of Learning. Educom Review, 29(4)

Wulf, K. (1996) Training via the Internet: Where Are We?. Training and Development, 50(5), 50-55.

\section{BIOGRAPHIES}

Dr. Baltasar Fernandez-Manjon is an assistant professor in Computer Science at the Complutense University of Madrid. He received his B.S. degree in physics and 
his Ph.D. degree in computer science from the Complutense University of Madrid. His research interest lie in the educational uses of computers and networks (specially, user modelling, intelligent help systems and multimedia applications).

Dr. Alfredo Fernandez-Valmayor is a professor in Computer Science at the Complutense University of Madrid. His research interest lies in the educational uses of computers, multimedia, and automatic text processing.

Mr. Antonio Navarro is a researcher at the Complutense University of Madrid. He received his B.S. degree in mathematics and now he is working in his Ph.D. about educational multimedia and automatic text processing. 